\title{
Tantalum Oxide Electron-Selective Heterocontacts for Silicon Photovoltaics and Photoelectrochemical Water Reduction
}

Yimao Wan, ${ }^{*}, \nabla, \dagger, \downarrow, \S \odot$ Siva Krishna Karuturi, ${ }^{\nabla, \|}$ Christian Samundsett, ${ }^{\dagger}$ James Bullock, $^{\ddagger, \S}$ Mark Hettick, ${ }^{\ddagger}, \S$ Di Yan, ${ }^{\dagger}$ Jun Peng, ${ }^{\dagger}$ Parvathala Reddy Narangari, " Sudha Mokkapati, ${ }^{\perp}$ Hark Hoe Tan," Chennupati Jagadish," Ali Javey, ${ }^{\ddagger, \S}$ and Andres Cuevas ${ }^{\dagger}$

${ }^{\dagger}$ Research School of Engineering, The Australian National University (ANU), Canberra, ACT 0200, Australia

${ }^{\ddagger}$ Department of Electrical Engineering and Computer Sciences, University of California, Berkeley, California 94720, United States

${ }^{\S}$ Materials Sciences Division, Lawrence Berkeley National Laboratory, Berkeley, California 94720, United States

"Department of Electronic Materials Engineering, Australian National University (ANU), Canberra, ACT 0200, Australia

${ }^{\perp}$ School of Physics and Astronomy, Cardiff University, Cardiff, Wales, U.K.

Supporting Information

ABSTRACT: Crystalline silicon (c-Si) solar cells have been dominating the photovoltaic (PV) market for decades, and c-Si based photoelectrochemical (PEC) cells are regarded as one of the most promising routes for water splitting and renewable production of hydrogen. In this work, we demonstrate a nanoscale tantalum oxide $\left(\mathrm{TaO}_{x}, \sim 6 \mathrm{~nm}\right)$ as an electron-selective heterocontact, simultaneously providing high-quality passivation to the silicon surface and effective transport of electrons to either an external circuit or a water-splitting catalyst. The PV application of $\mathrm{TaO}_{x}$ is demonstrated by a proof-of-concept device having a conversion efficiency of $19.1 \%$. In addition, the PEC application is demonstrated by a photon-to-current efficiency (with additional applied bias) of 7.7\%. These results represent a $2 \%$ and $3.8 \%$ absolute enhancement over control devices without a $\mathrm{TaO}_{x}$ interlayer, respectively. The methods presented in this Letter are not limited to c-Si based devices and can be viewed as a more general approach to the interface engineering of optoelectronic and photoelectrochemical applications.

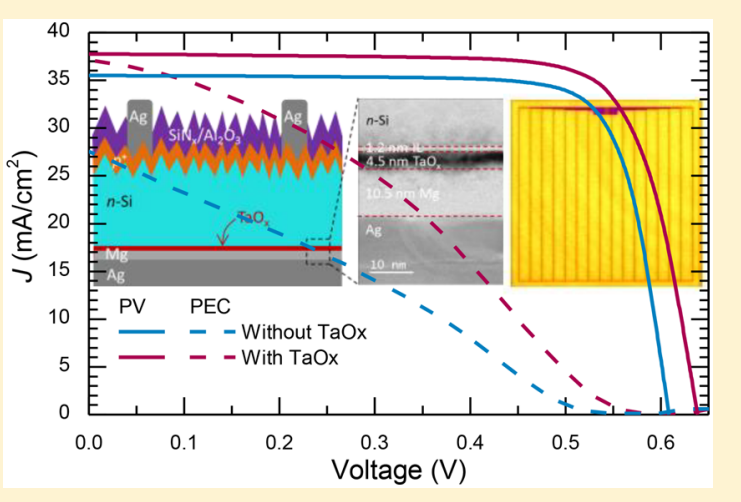

$\mathrm{W}$ ith increasing concerns over rising global energy demand and environmental sustainability, the development of renewable energy technologies is of great importance to the continuation of socio-economic development. There is growing consensus that significant contributions can come from the conversion of solar energy into electricity using photovoltaic (PV) cells ${ }^{1}$ and/or into chemical energy using photoelectrochemical (PEC) devices. ${ }^{2}$ With a $90 \%$ share of global PV market, crystalline silicon $(\mathrm{c}-\mathrm{Si})$ is unequivocally the most important photovoltaic technology. Because of its high abundance in the earth's crust and industrial maturity, c-Si based systems have also received significant attention for PEC applications. ${ }^{3-7}$ Both PV and PEC devices rely on the same central working principle: photogenerated electrons and holes are separately collected at the two opposite terminals of the device. A common method to achieve this function is via doping (i.e., boron-doped $p^{+}$or phosphorus- doped $n^{+}$) the near-surface regions of the silicon wafer or thin silicon films deposited on it. ${ }^{8-10}$

Another attractive approach to separately collect the two types of charge carriers is to use metal-insulator-semiconductor (MIS) passivated heterocontact structures. A typical MIS heterocontact is composed of a metal layer positioned on the outer surface of an oxide passivated semiconductor. To be able to function as passivated heterocontacts, the oxide interlayers have to fulfill several critical requirements: (i) effective passivation of defects typically present at the silicon surface, (ii) efficient transport of only one carrier type (for example electrons) and effective blocking of the other carrier (for example holes) in their path from the silicon photon absorber to the outer metal terminals, and (iii) simple synthesis

Received: November 20, 2017

Accepted: December 8, 2017

Published: December 8, 2017 
method at low thermal budget. Specific to PEC applications, the oxide interlayers also need to be chemically robust to survive in a very harsh aqueous environment for extended operation.

Ultrathin tunnelling silicon dioxide $\left(\mathrm{SiO}_{2}\right.$, typically $\left.\sim 1.5 \mathrm{~nm}\right)$ is one of the most explored oxides in heterocontacts for both $\mathrm{PV}^{11,12}$ and PEC ${ }^{4,6,13,14}$ applications because of its reasonable passivation quality and effective transport of carriers (i.e., permits the transport of majority carriers together with a low recombination of minority carriers) $)^{11,12}$ and its stability over a wide range of $\mathrm{pH}$ and chemical potentials. ${ }^{15}$ However, the highly insulating bulk properties of $\mathrm{SiO}_{2}$ lead to inhibition of charge transport through the MIS heterocontacts, restricting conduction to tunnelling, or through pinholes. Furthermore, the high-quality ultrathin $\mathrm{SiO}_{2}$ places stringent requirements in film thickness control and process environment purity. Profound efforts have been recently devoted to exploring alternatives to $\mathrm{SiO}_{2}$ as the interlayer to form passivated carrierselective heterocontacts on c-Si wafers, such as metal salts ${ }^{16,17}$ and oxides ${ }^{18}$ and organic polymers. ${ }^{19,20}$

Another group of candidate materials for MIS passivated heterocontacts are the transition-metal oxides, some of which possess almost all critical characteristics for carrier-selective contacts, including a wide range of work functions, semiconducting properties, and high transparency to sunlight. ${ }^{21}$ Research on the incorporation of transition-metal oxides into Si-based PV and PEC devices is very recent. High work function oxides such as molybdenum oxide, tungsten oxide, vanadium oxide, and cuprous oxide have been explored as holeselective contacts in silicon solar cells, ${ }^{22-27}$ whereas nickel oxide and cobalt oxide have been studied as effective photoanodes for water oxidation. ${ }^{28-30}$ Remarkably, defective titanium oxide has also been reported to promote hole transport for efficient water oxidation. ${ }^{31,32}$ In contrast, research on transition-metal oxides as electron-selective contacts on crystalline silicon is scarce, with titanium oxide and zinc oxide so far the only transition-metal oxides reported on $\mathrm{Si}$ solar cells $^{33-35}$ and strontium titanium oxide as Si photocathodes for water reduction. ${ }^{13}$

Although it has not received as much attention yet, tantalum oxide $\left(\mathrm{TaO}_{x}\right)$ is a promising material for electron-selective contacts to silicon because of (i) small conduction band offset, ${ }^{21,36}$ which allows electrons from the silicon conduction band to flow through $\mathrm{TaO}_{x}$; (ii) large valence band offset $(\sim 2.9$ $\mathrm{eV}),{ }^{21,36}$ which provides a barrier to prevent holes in the silicon valence band from flowing to the oxide and further to the metal cathode; and (iii) recently demonstrated effective passivation of silicon surface defects. ${ }^{37,38}$ In addition, $\mathrm{TaO}_{x}$ possesses high thermal and chemical stability under various $\mathrm{pH}$ conditions, ${ }^{39-42}$ making it a sensible choice for PEC applications. In this work, we report a nanoscale $\mathrm{TaO}_{x}$ film $(\sim 6 \mathrm{~nm})$ as a robust passivated electron-selective heterocontact for $\mathrm{c}-\mathrm{Si}$ based photovoltaics and photoeletrochemical water reduction for the first time, demonstrating significantly improved solar-toelectricity and solar-to-hydrogen conversion efficiencies.

We investigate the optoelectronic properties of $\mathrm{TaO}_{x}$ films synthesized by atomic layer deposition (ALD) and hydrogenated by plasma-enhanced chemical vapor deposited (PECVD) silicon nitride $\left(\mathrm{SiN}_{x}\right)$, including core-level, valence band, work function, contact resistivity, and surface passivation properties. We then demonstrate, for the first time, the application of hydrogenated $\mathrm{TaO}_{x}$ to Si-based PV and PEC devices for the generation of electricity and hydrogen, achieving a solar-to-electricity efficiency of $19.1 \%$ and applied bias photon-to-current efficiency of $7.7 \%$, which correspond to $2 \%$ and $3.8 \%$ absolute enhancement, respectively, over control devices without a $\mathrm{TaO}_{x}$ interlayer.

$\mathrm{X}$-ray photoelectron spectroscopy (XPS) was employed to reveal $\mathrm{TaO}_{x}$ electronic properties, including stoichiometry, valence band, and work function, which are critical to the transport of electrons. The results are shown in Figure 1. While

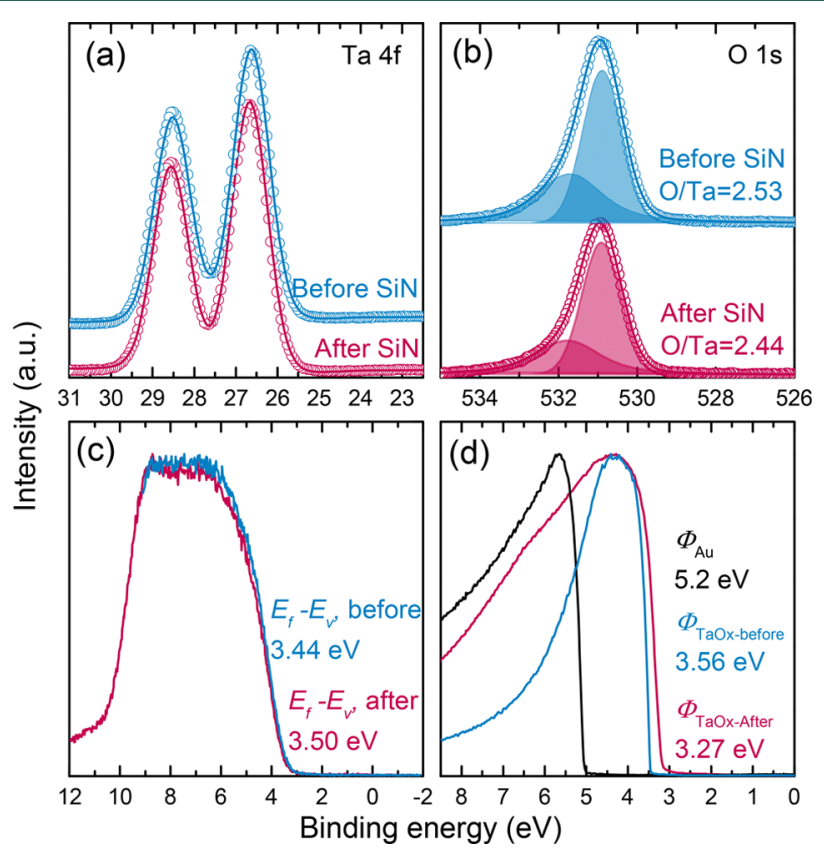

Figure 1. Electronic characterizations of atomic layer deposited $\mathrm{TaO}_{x}$ films before and after hydrogenation. Panels a and $\mathrm{b}$ present the core-level spectrum of Ta $4 \mathrm{f}$ and $\mathrm{O} 1 \mathrm{~s}$, respectively. Panel $\mathrm{c}$ shows the valence band spectrum, and panel d shows the secondary electron cutoff spectrum with a gold $(\mathrm{Au})$ reference.

the Ta 4f spectra shows typical doublet peaks located at 26.5 and $28.5 \mathrm{eV}$ for Ta $4 \mathrm{f} 7 / 2$ and $\mathrm{Ta} 4 \mathrm{f} 5 / 2$, respectively, ${ }^{43}$ the $\mathrm{O}$ 1s spectra can be fitted with two Gaussian components with peaks positioned at 530.8 and $532 \mathrm{eV}$, respectively. The small peak located at $532 \mathrm{eV}$ is usually attributed to peroxide $\mathrm{O}_{2}{ }^{2-}$, although sometimes it can also be due to surface contamination. ${ }^{43}$ The component with lower binding energy peaked at $530.8 \mathrm{eV}$ is from Ta-O binding. ${ }^{43}$ Notably, the peak positions for both Ta $4 \mathrm{f}$ and $\mathrm{O} 1 \mathrm{~s}$ are observed to be the same for the $\mathrm{TaO}_{x}$ film before and after $\mathrm{SiN}_{x}$ hydrogenation. However, we can notice a slight reduction in the core level peak areas of $\mathrm{O} 1 \mathrm{~s}$ spectra after $\mathrm{SiN}_{x}$ hydrogenation. The extracted $\mathrm{TaO}_{x}$ film stoichiometry based on core level peak areas is also included in Figure $1 \mathrm{~b}$. The result shows the as-deposited $\mathrm{TaO}_{x}$ has an $\mathrm{O}$ to Ta atomic fraction of 2.53, and the $\mathrm{TaO}_{x}$ after $\mathrm{SiN}_{x}$ hydrogenation has an $\mathrm{O}$ to $\mathrm{Ta}$ atomic fraction of 2.44 , implying the hydrogenation process makes the $\mathrm{TaO}_{x}$ film slightly more substoichiometric.

Figure 1c presents the valence band spectrum of the two $\mathrm{TaO}_{x}$ films, showing no significant difference ( $<2 \%$ change). Although the $\mathrm{TaO}_{x}$ film after $\mathrm{SiN}_{x}$ hydrogenation is more metallic, no defect band in the band gap can be seen, suggesting negligible amount of filled states at the Fermi level. Furthermore, the XPS secondary electron cutoff result shows that the $\mathrm{TaO}_{x}$ film after $\mathrm{SiN}_{x}$ hydrogenation has a work function of $3.27 \mathrm{eV}$, approximately $0.3 \mathrm{eV}$ lower than that of as- 
deposited $\mathrm{TaO}_{x}$. The lower work function can be attributable to the lower cation oxidation state that was revealed by the core level measurements. As proposed in ref 44, the lower cation oxidation state would result in lower work function, because lower oxidation-state cations are less electronegative than higher oxidation-state cations. The measured work function of the $\mathrm{TaO}_{x}$ films is significantly lower than that of $\mathrm{TiO}_{2}$ presented in the literature (i.e., $\sim 4.2 \mathrm{eV}$ ). ${ }^{45,46}$ The reduced work function is expected to promote downward band-bending inside the silicon wafer drawing electrons to the surface and consequently improving electron transport.

As mentioned above, $\mathrm{TaO}_{x}$ combines a high valence band offset, creating a potential barrier to hole transport; nevertheless, holes can still flow to the interface between $\mathrm{TaO}_{x}$ and $\mathrm{Si}$, unless it is perfectly passivated. Therefore, the carrier selectivity of an $n$-Si/ $\mathrm{TaO}_{x}$ heterostructure needs to be evaluated by characterizing not only the contact resistivity, $\rho_{\mathcal{O}}$ but also the surface recombination velocity. A highly selective contact is achieved through a simultaneous reduction in recombination and contact resistance. One of the most straightforward techniques to probe the recombination properties of c-Si surfaces is by measuring the effective minority carrier lifetime $\left(\tau_{\text {eff }}\right)$ of symmetrically film-passivated wafers in a contactless fashion, ${ }^{47}$ as illustrated in Figure $\mathrm{S} 1$ in the Supporting Information.

Figure $2 \mathrm{a}$ depicts the quality of surface passivation provided by $6 \mathrm{~nm} \mathrm{TaO}_{x}$ films before and after $\mathrm{SiN}_{x}$ hydrogenation by plotting the injection-dependent effective carrier lifetime $\tau_{\text {eff }}(\Delta n)$ of $n$-type undiffused c-Si samples. As can be seen, the as-deposited $\mathrm{TaO}_{x}$ film (i.e., before hydrogenation) provides a poor passivation to silicon surfaces, with $\tau_{\text {eff }}$ of
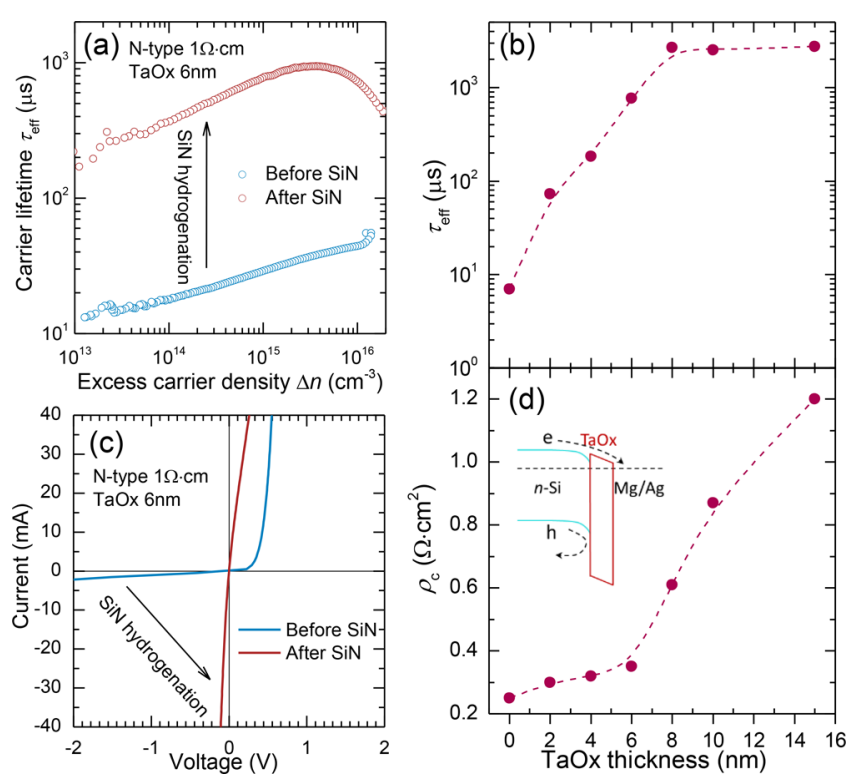

Figure 2. Carrier selectivity characterizations of $\mathrm{TaO}_{x}$ passivated electron heterocontacts to $n$-type c-Si. Panel a presents the effective carrier lifetime $\tau_{\text {eff }}$ versus excess carrier density $\Delta n$ for c-Si passivated with $\mathrm{TaO}_{x}$ films before and after $\mathrm{SiN}_{x}$ hydrogenation. Panel $b$ presents the effect of hydrogenated $\mathrm{TaO}_{x}$ film thickness on $\tau_{\text {eff }}$ Panel c presents representative $I-V$ measurements of $\mathrm{TaO}_{x}$ samples before and after $\mathrm{SiN}_{x}$ hydrogenation. Panel $\mathrm{d}$ shows the effect of hydrogenated $\mathrm{TaO}_{x}$ film thickness on the contact resistivity $\rho_{c}$. The inset in panel $\mathrm{d}$ shows a schematic illustration of the band diagram with hydrogenated $\mathrm{TaO}_{x}$. $\sim 30 \mu \mathrm{s}$ at an excess carrier density $\Delta n=10^{15} \mathrm{~cm}^{-3}$. The level of surface passivation is improved more than 1 order of magnitude upon a $\mathrm{SiN}_{x}$ hydrogenation treatment, resulting in $\tau_{\text {eff }}$ as high as $\sim 650 \mu$ s at $\Delta n=10^{15} \mathrm{~cm}^{-3}$, corresponding to a recombination current density $J_{0}$ of $22.5 \mathrm{fA} / \mathrm{cm}^{2}$ and an implied open-circuit voltage $\left(V_{\mathrm{OC}}\right)$ of $690 \mathrm{mV}$. Figure $2 \mathrm{~b}$ shows that the passivation quality of hydrogenated $\mathrm{TaO}_{x}$ films depends strongly on the film thickness; the corresponding injection-dependent lifetime curves are shown in detail as Figure $\mathrm{S} 1 \mathrm{~b}$. As the $\mathrm{TaO}_{x}$ thickness increases, $\tau_{\text {eff }}$ first increases and then tends to plateau at $8 \mathrm{~nm}$ $\mathrm{TaO}_{x}$. The highest lifetime achieved at $\Delta n=10^{15} \mathrm{~cm}^{-3}$ is $\sim 2.7$ ms. The substantial enhancement in passivation upon $\operatorname{SiN}_{x}$ hydrogenation is mainly attributable to the hydrogenation of defects at the $\mathrm{TaO}_{x} / \mathrm{c}$-Si interface during the deposition of $\mathrm{SiN}_{x}{ }^{38}$

The contact resistivity $\rho_{\mathrm{c}}$ of $\mathrm{TaO}_{x}$ to $n$-type c-Si was measured using the method devised by Cox and Strack. ${ }^{48}$ The test structure is schematically depicted in Figure S2a. Low work function metal $\mathrm{Mg}$ was employed here to further enhance the electron transport, as demonstrated in previous work. ${ }^{49}$ Figure 2 c shows a representative $I-V$ measurement of $\mathrm{TaO}_{x}$ samples before and after the $\mathrm{SiN}_{x}$ hydrogenation treatment. As can be seen, the sample before $\mathrm{SiN}_{x}$ hydrogenation exhibits severe rectifying behavior and a high contact resistivity. By contrast, the contact behavior of the $\mathrm{TaO}_{x}(\sim 6 \mathrm{~nm})$ film is enhanced dramatically upon $\mathrm{SiN}_{x}$ hydrogenation, resulting in an Ohmic contact to the $n$-type $\mathrm{Si}$ substrate. The full series of $I-V$ measurements are shown in Figure S2b; from that series, the $\rho_{\mathrm{c}}$ for the heterocontact with $\sim 6 \mathrm{~nm}$ hydrogenated $\mathrm{TaO}_{x}$ is determined to be $\sim 0.35 \Omega \cdot \mathrm{cm}^{2}$, which is sufficiently low to function as a full area heterocontact for $n$-type silicon PV and PEC devices. The good electron transport provided by the hydrogenated $\mathrm{TaO}_{x}$ on $n$-type $\mathrm{Si}$ can be attributed to (i) a reduced work function and/or (ii) Fermi-level depinning as a consequence of the passivation of interface states between $\mathrm{TaO}_{x}$ and the silicon substrate. The dependence of contact resistivity on $\mathrm{TaO}_{x}$ thickness is depicted in Figure 2d, exhibiting a slow increase in $\rho_{\mathrm{c}}$ and then a sharp increase when $\mathrm{TaO}_{x}$ is beyond $6 \mathrm{~nm}$. Indeed, when $\mathrm{TaO}_{x}$ thickness exceeds $10 \mathrm{~nm}$, the contact behaves in a rectifying fashion even after the $\mathrm{SiN}_{x}$ hydrogenation. The increasing trend in both $\tau_{\text {eff }}$ and $\rho_{\mathrm{c}}$ creates a trade-off between surface passivation and contact resistivity provided by the $\mathrm{TaO}_{x}$ film. To resolve this trade-off, we fabricate completed silicon solar cells in order to find the optimum $\mathrm{TaO}_{x}$ film thickness for maximizing the carrier selectivity.

Upon quantifying the carrier selectivity of $\mathrm{TaO}_{x}$, we can now perform a comparison to state-of-the-art electron-selective contacts reported in the literature. Table S1 in the Supporting Information summarizes the performance parameters of the various electron-selective contacts, including work function, contact resistivity, and recombination current density. Compared to the lowest work function materials, such as alkali and alkaline earth metals or salts, $\mathrm{TaO}_{x}$ itself can provide an effective passivation of the silicon surface, with a recombination current density of just $J_{0} \sim 22.5 \mathrm{fA} / \mathrm{cm}^{2}$. In contrast, to achieve low recombination in the case of electron-selective contacts based on alkali or alkaline earth metal salts, it is necessary to insert an additional passivation layer, such as amorphous silicon, without significant compromise in the contact resistivity. ${ }^{50}$ The surface passivation quality by $\mathrm{TaO}_{x}$ is slightly better than the recently reported $\mathrm{SiO}_{2} / \mathrm{TiO}_{2}$ stack which has enabled an efficiency of $22.1 \%$ silicon solar cells, ${ }^{51}$ demonstrat- 
(a)

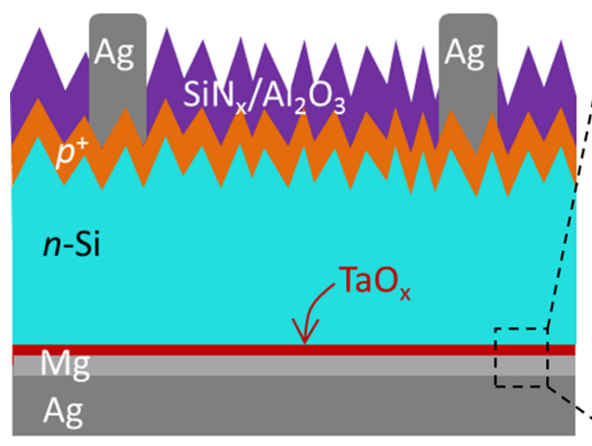

(d)

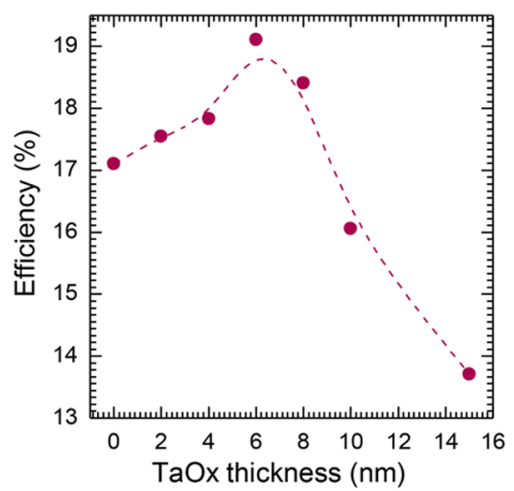

(b)

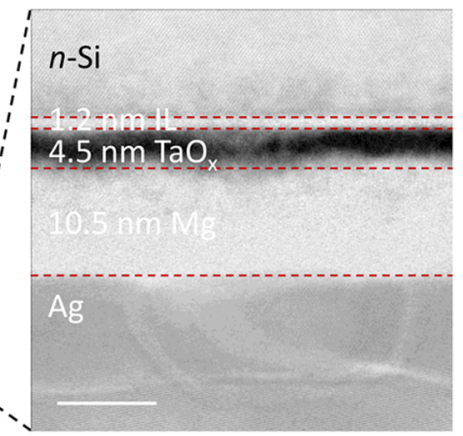

(e)

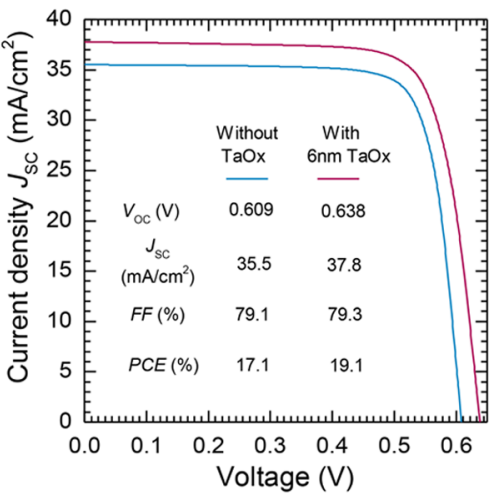

(c)

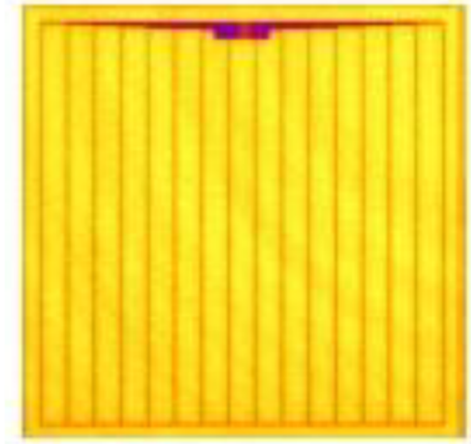

(f)

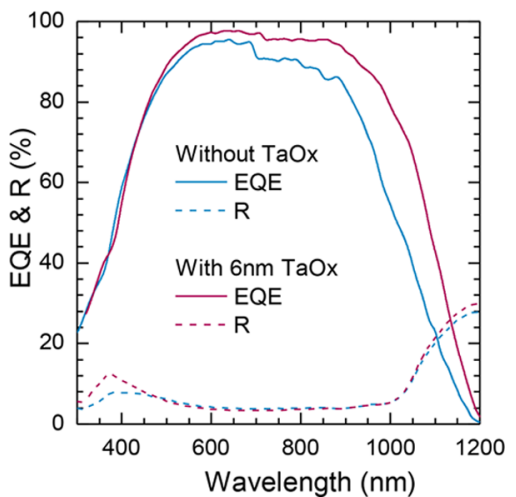

Figure 3. PV device performance with a full-area rear $\mathrm{TaO}_{x}$ passivated electron heterocontact. (a) Schematic cross-section of an $n$-type silicon solar cell featuring a full-area $\mathrm{TaO}_{x} / \mathrm{Mg} / \mathrm{Ag}$ rear contact. (b) Cross-sectional transmission electron microscopy (TEM) image of the rear heterocontacts. (c) Photoluminescence image of the complete solar cell. (d) Dependence of cell efficiency on $\mathrm{TaO}_{x}$ film thickness. (e) Light $J-V$ curves and electrical parameters of cells with and without a $\sim 6 \mathrm{~nm} \mathrm{TaO}$ interlayer. (f) Spectral response (external quantum efficiency and reflectance) of the two cells.

ing a high potential to achieve high-efficiency solar cells by using $\mathrm{TaO}_{x}$-based electron-selective heterocontacts. It is notable, however, that in our experiments it has been necessary to hydrogenate the $\mathrm{TaO}_{x} / \mathrm{Si}$ interface by depositing a sacrificial layer of $\mathrm{SiN}_{x}$; it should be possible, however, to perform such hydrogenation by other simpler means, such as hydrogen plasma treatment. ${ }^{52} \mathrm{~A}$ distinctive feature is that, among all the electron-selective contacts in Table $\mathrm{S} 1, \mathrm{TaO}_{x}$ exhibits the highest stability against chemical solutions, making it a sensible choice for photoelectrochemical applications.

The complete silicon solar cells feature full area one dimensional $\mathrm{TaO}_{x}$ passivated heterocontacts and are schematically depicted in Figure 3a. Figure $3 \mathrm{~b}$ presents a cross-sectional transmission electron micrograph of the rear heterocontacts, showing the $\sim 6 \mathrm{~nm} \mathrm{TaO}$ consists of $\sim 1.2 \mathrm{~nm}$ interfacial layer and $\sim 4.5 \mathrm{~nm} \mathrm{TaO}_{x}$. The interfacial layer may result from exposure to ambient after deionized water rinse or water precursor during the first few ALD cycles. A photoluminescence image of the complete solar cells (Figure 3c) employing a $1025 \mathrm{~nm}$ short-pass filter shows the cell surfaces had uniform optical, passivating, and contact behaviors. Figure $3 \mathrm{~d}$ shows the solar cell power conversion efficiency as a function of $\mathrm{TaO}_{x}$ film thickness [detailed electrical parameters for these cells $\left(V_{\mathrm{OC}}, J_{\mathrm{SC}}\right.$, and $\left.\mathrm{FF}\right)$ are presented in Figure $\mathrm{S} 3$ and Table S2]. As can be seen, the efficiency exhibits a strong dependence on $\mathrm{TaO}_{x}$ film thickness, with a maximum at a thickness of $6 \mathrm{~nm}$. As $\mathrm{TaO}_{x}$ film thickness increases, the efficiency first increases primarily because of an increase in surface passivation quality and therefore an enhancement in $V_{\mathrm{OC}}$ and then decreases after $6 \mathrm{~nm}$ mainly because of a high contact resistivity imposed by the thicker $\mathrm{TaO}_{x}$ film and therefore a reduction in FF. These solar cells trends are consistent with the carrier selectivity results presented in Figure 2.

The illuminated $J-V$ curve for the champion cell with $6 \mathrm{~nm}$ $\mathrm{TaO}_{x}$ is plotted in Figure 3e, which also includes the reference cell without a $\mathrm{TaO}_{x}$ film. It can be seen that all cell parameters were improved drastically by inserting the $6 \mathrm{~nm}$ thick $\mathrm{TaO}_{x}$ layer, yielding a $19.1 \%$ solar-to-electricity conversion efficiency, which is $2 \%$ absolute higher than the $17.1 \%$ reference cell. Compared to the reference cell with metal directly on silicon, an absolute gain of $29 \mathrm{mV}$ in open-circuit voltage was obtained, primarily because of the improved surface passivation provided by the hydrogenated $\mathrm{TaO}_{x}$ film. The suppression of the recombination at rear silicon surfaces also leads to a gain of 2.3 $\mathrm{mA} / \mathrm{cm}^{2}$ in short-circuit current. It is interesting to note that the fill factor is negligibly affected by the insertion of $6 \mathrm{~nm}$ $\mathrm{TaO}_{x}$, despite a slight increase in contact resistivity. It is worth mentioning that the obtained $V_{\mathrm{OC}}(638 \mathrm{mV})$ is significantly lower than the implied $V_{\mathrm{OC}}$ from the lifetime sample $(\sim 690$ $\mathrm{mV}$ ). One tentative cause for this behavior is the damage to surface passivation during $\mathrm{Mg}$ metal evaporation, a behavior that has also been observed for the case of amorphous silicon. ${ }^{49}$ A possible solution to mitigate this damage would be the use of other low work function materials such as lithium or magnesium fluorides. ${ }^{16,17}$ The spectral response plotted in 
Figure $3 \mathrm{f}$ shows a similar reflectance but a significant enhancement in the external quantum efficiency (EQE) at long wavelengths (i.e., range from $\sim 900-1200 \mathrm{~nm}$ ) for the cell with the $\mathrm{TaO}_{x}$ heterocontact, due to the passivation of the rear surface by the $\mathrm{TaO}_{x}$ layer. This is consistent with the abovementioned improvements in effective lifetime, voltage, and current and provides additional evidence of the enhancements realized from $\mathrm{TaO}_{x}$ passivation.

Finally, the concept of electron transport by hydrogenated $\mathrm{TaO}_{x}$ is also demonstrated for photoelectrochemical hydrogen evolution. The electron-selective heterocontact was prepared by replacing the $\mathrm{Mg} / \mathrm{Ag}$ metal stack, which we found not to be chemically stable, with $5 \mathrm{~nm}$ Ti metal and $5 \mathrm{~nm}$ Pt catalyst on a $\sim 6 \mathrm{~nm} \mathrm{TaO}_{x}$ film, to enable stable and efficient hydrogen evolution reactions, as shown in Figure $4 \mathrm{a}$. Ti metal with its (a)

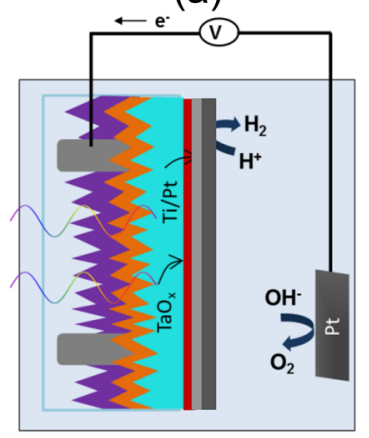

(c)

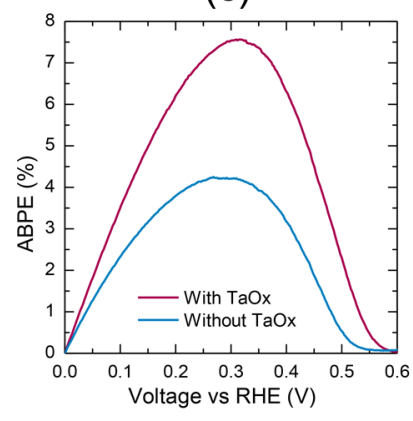

(b)

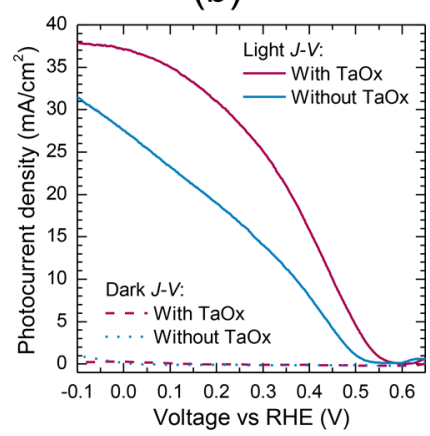

(d)

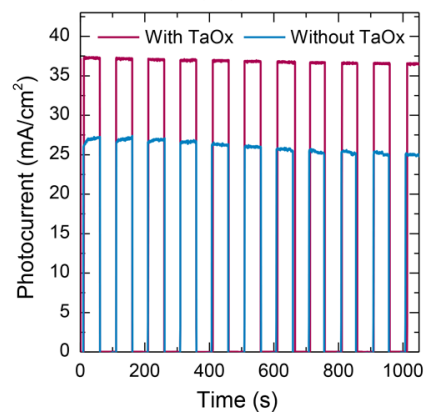

Figure 4. PEC device results with $\mathrm{TaO}_{x}$ passivated electron heterocontacts. Panel a illustrates the schematic of an $n$-type silicon photocathode using $\mathrm{TaO}_{x}$ passivated electron heterocontacts. Panel b presents the photoelectrochemical current-voltage curves of $\mathrm{Si}$ photocathodes with and without $\sim 6 \mathrm{~nm} \mathrm{TaO}$, interlayer. Panel c shows applied bias photon-to-current efficiency (ABPE). Panel d shows amperometric current-time curves of $\mathrm{Si}$ photocathodes at $0 \mathrm{~V}$ vs the reversible hydrogen electrode (RHE).

relatively low work function $\left(\sim 4.2 \mathrm{eV}^{53}\right)$ assists in reducing the barrier between $\mathrm{TaO}_{x}$ and $\mathrm{Pt}$, improving the photovoltage over a device with $\mathrm{Pt}$ only. ${ }^{4}$ When the silicon device is illuminated, photogenerated minority carriers (holes in this case) flow toward the illuminated $p^{+}$region at the front surface, whereas the majority carriers (electrons in this case) are transported through the $\mathrm{TaO}_{x}$ interlayer to the outer $\mathrm{Ti} / \mathrm{Pt}$ catalyst, where the hydrogen evolution reaction takes place.

For comparison, a control PEC device was prepared without a $\mathrm{TaO}_{x}$ film. Figure $4 \mathrm{~b}$ compares the photoelectrochemical performance of these two PEC devices, with and without $\mathrm{TaO}_{x}$. The control device shows an onset potential of $525 \mathrm{mV}$ for water reduction, whereas the photoelectrode with $\sim 6 \mathrm{~nm}$ of
$\mathrm{TaO}_{x}$ showed a more positive onset potential of $565 \mathrm{mV}$. The photocurrent density of the device with a $\mathrm{TaO}_{x}$ passivated electrode reaches $37.1 \mathrm{~mA} / \mathrm{cm}^{2}$ at $0 \mathrm{~V}$ vs RHE, compared to the $27.5 \mathrm{~mA} / \mathrm{cm}^{2}$ obtained for the control electrode without $\mathrm{TaO}_{x}$. The significant enhancement in both photocurrent and photovoltage is consistent with the improved photovoltaic characteristics shown in Figure 3, and can be attributed to the role of $\mathrm{TaO}_{x}$ in suppressing carrier recombination at the rear surface while maintaining efficient electron transport.

To evaluate the improvement in photoelectrochemical performance with $\mathrm{TaO}_{x}$ layer, applied bias photon-to-current efficiency (ABPE) has been calculated using the method reported previously. ${ }^{54}$ It is noted that $A B P E$ values derived from three-electrode measurements do not include the losses that might be present at the counter electrode in a twoelectrode system. ABPE for $\mathrm{TaO}_{x}$ passivated electrode has been measured to reach $7.7 \%$, which is almost double that of the control electrode without $\mathrm{TaO}_{x}$ interlayer. However, this PEC conversion efficiency is well below the $19.1 \%$ achieved by the best photovoltaic device incorporating a $\mathrm{TaO}_{x}$ interlayer, mainly because of lower photovoltage and FF. Beyond the thermodynamic energy loss associated with the water reduction electrolyte, this disparity can be largely attributed to an unfavorable energy band alignment in the $\mathrm{Ti} / \mathrm{TaO}_{x} / \mathrm{Si}$ photocathode, compared to the $\mathrm{Mg} / \mathrm{TaO}_{x} / \mathrm{Si}$ contact architecture used in the PV devices. The use of Ti instead of $\mathrm{Mg}$ can potentially lead to a higher barrier height for electron transport and therefore cause significant loss in the electron contact resistivity. Nevertheless, these experiments have demonstrated, for the first time, that hydrogentated $\mathrm{TaO}_{x}$ is capable of providing passivation of the silicon surface and, simultaneously, transport efficiently the electrons for PEC water reduction.

As the electrodes used for water splitting must endure a highly corrosive and reducing environment, stability tests have been carried out to evaluate the photoresponse and photostability. As shown in Figure 4d, the reference electrode exhibited a slight drop in photocurrent with time, while the photoelectrode with $\mathrm{TaO}_{x}$ film showed a highly stable photoresponse. It is known that $\mathrm{c}-\mathrm{Si}$ is stable in acid environments while it is highly unstable in oxidizing conditions. The instability of the control electrode without $\mathrm{TaO}_{x}$ interlayer is likely attributed to the lack of hole-blocking effect at the interface, inducing possible self-oxidizing conditions. The longterm stability of the $\mathrm{TaO}_{x}$-based electrode is further confirmed in $1 \mathrm{M}$ hydrochloric acid solution for $2 \mathrm{~h}$ of continuous operation (see Figure S4).

We have successfully demonstrated a hydrogenated $\mathrm{TaO}_{x}$ $(\sim 6 \mathrm{~nm})$ electron-selective heterocontact that simultaneously provides high quality passivation of the silicon surface (i.e., lifetime $\sim 650 \mu \mathrm{s}$ ) and effective transport of electrons (i.e., contact resistivity $\left.\sim 0.35 \Omega \cdot \mathrm{cm}^{2}\right)$. The XPS measurements revealed the hydrogenation induced by PECVD $\mathrm{SiN}_{x}$ resulted in reduction in $\mathrm{TaO}_{x}$ stoichiometry and work function, making $\mathrm{TaO}_{x}$ film favorable for electron contacts. We have achieved a solar-to-electricity efficiency of $19.1 \%$ and a solar-to-hydrogen efficiency of $7.7 \%$, which correspond to a $2 \%$ and $3.8 \%$ absolute enhancement, respectively, over the control devices without a $\mathrm{TaO}_{x}$ interlayer. These findings related to tantalum oxide are not limited to a silicon-based device platform; more generally, they indicate a generic pathway for interface engineering of optoelectronic and photoelectrochemical applications. 


\section{EXPERIMENTAL METHODS}

The $\mathrm{TaO}_{x}$ films were deposited by a thermal ALD reactor (Picosun, R200 Advanced) using Tantalum Ethoxide precursor at $250{ }^{\circ} \mathrm{C}$ and had a corresponding growth rate of $0.35 \AA /$ cycle as measured by ex-situ spectroscopic ellipsometry (J.A. Woolam M2000 ellipsometer). The hydrogenation of $\mathrm{TaO}_{x}$ film was realized by capping PECVD $\mathrm{SiN}_{x}$ atop $\mathrm{TaO}_{x}$ and then stripping off the $\mathrm{SiN}_{x}$ layer by $1 \%$ hydrofluoric acid, taking advantage of the exceptional chemical stability of $\mathrm{TaO}_{x}$ film under various $\mathrm{pH}$ conditions. PECVD $\mathrm{SiN}_{x}$ has been well-known in providing hydrogen to manipulate bulk and interfacial properties of a range of materials such as silicon. ${ }^{55}$ The capping $\mathrm{SiN}_{x}$ layer was deposited in a microwave/radio frequency PECVD reactor (AK400, Roth \& Rau), ${ }^{56}$ having a thickness of $85 \mathrm{~nm}$ and a refractive index of $\sim 1.9$ at $632 \mathrm{~nm}$.

Further experimental details are given in the Supporting Information.

\section{ASSOCIATED CONTENT}

\section{S Supporting Information}

The Supporting Information is available free of charge on the ACS Publications website at DOI: 10.1021/acsenergylett.7b01153.

Characterization methods including X-ray photoelectron spectroscopy, carrier lifetime, and contact resistivity; fabrication and characterization details of silicon PV and PEC devices (PDF)

\section{AUTHOR INFORMATION}

\section{Corresponding Author}

*E-mail: yimao.wan@anu.edu.au.

\section{ORCID}

Yimao Wan: 0000-0003-2999-2464

\section{Author Contributions}

Y.W. supervised the project. Y.W. and S.K.K. conceived the idea. Y.W. and C.S. carried out the material development, device fabrication, characterization, and analysis. Y.W., S.K.K., and P.R.N. conducted the water-splitting experiment. J.B. and M.H. assisted with XPS characterization and analysis. D.Y. and J.P. assisted with PV device fabrication and characterization. S.M., H.H.T., C.J., A.J., and A.C. discussed the results. The manuscript was mainly written and revised by Y.W. and S.K.K. All authors commented on the manuscript.

\section{Author Contributions}

${ }^{\nabla}$ Y.W. and S.K.K. contributed equally to this work.

\section{Notes}

The authors declare no competing financial interest.

\section{ACKNOWLEDGMENTS}

This work was supported by the Australian Government through the Australian Research Council (ARC). Some facilities at the Australian National Fabrication Facility and Centre for Advanced Microscopy at ANU were used. XPS characterization was performed at the Joint Center for Artificial Photosynthesis, supported through the Office of Science of the U.S. Department of Energy under Award Number DESC0004993. A.J., M.H., and J.B. acknowledge funding from the Bay Area Photovoltaics Consortium (BAPVC).

\section{REFERENCES}

(1) Creutzig, F.; Agoston, P.; Goldschmidt, J. C.; Luderer, G.; Nemet, G.; Pietzcker, R. C. The underestimated potential of solar energy to mitigate climate change. Nature Energy 2017, 2, 17140.

(2) Walter, M. G.; Warren, E. L.; McKone, J. R.; Boettcher, S. W.; Mi, Q.; Santori, E. A.; Lewis, N. S. Solar Water Splitting Cells. Chem. Rev. 2010, 110, 6446-6473.

(3) Sun, K.; Shen, S.; Liang, Y.; Burrows, P. E.; Mao, S. S.; Wang, D. Enabling Silicon for Solar-Fuel Production. Chem. Rev. 2014, 114, 8662-8719.

(4) Esposito, D. V.; Levin, I.; Moffat, T. P.; Talin, A. A. H2 evolution at Si-based metal-insulator-semiconductor photoelectrodes enhanced by inversion channel charge collection and $\mathrm{H}$ spillover. Nat. Mater. 2013, 12, 562-568.

(5) Reece, S. Y.; Hamel, J. A.; Sung, K.; Jarvi, T. D.; Esswein, A. J.; Pijpers, J. J. H.; Nocera, D. G. Wireless Solar Water Splitting Using Silicon-Based Semiconductors and Earth-Abundant Catalysts. Science 2011, 334, 645-648.

(6) Ji, L.; Hsu, H.-Y.; Li, X.; Huang, K.; Zhang, Y.; Lee, J. C.; Bard, A. J.; Yu, E. T. Localized dielectric breakdown and antireflection coating in metal-oxide-semiconductor photoelectrodes. Nat. Mater. 2017, 16, $127-131$.

(7) Zhou, X.; Liu, R.; Sun, K.; Papadantonakis, K. M.; Brunschwig, B. S.; Lewis, N. S. $570 \mathrm{mV}$ photovoltage, stabilized $\mathrm{n}-\mathrm{Si} / \mathrm{CoOx}$ heterojunction photoanodes fabricated using atomic layer deposition. Energy Environ. Sci. 2016, 9, 892-897.

(8) Zhao, J. Recent advances of high-efficiency single crystalline silicon solar cells in processing technologies and substrate materials. Sol. Energy Mater. Sol. Cells 2004, 82, 53-64.

(9) Glunz, S.; Feldmann, F.; Richter, A.; Bivour, M.; Reichel, C.; Steinkemper, H.; Benick, J.; Hermle, M. The irresistible charm of a simple current flow pattern - approaching $25 \%$ with a solar cell featuring a full-area back contact. In 31st European Photovoltaic Solar Energy Conference and Exhibition, Hamburg, Germany, September, 2015.

(10) Masuko, K.; Shigematsu, M.; Hashiguchi, T.; Fujishima, D.; Kai, M.; Yoshimura, N.; Yamaguchi, T.; Ichihashi, Y.; Mishima, T.; Matsubara, N.; et al. Achievement of More Than 25\% Conversion Efficiency With Crystalline Silicon Heterojunction Solar Cell. Photovoltaics, IEEE Journal of 2014, 4, 1433-1435.

(11) Godfrey, R. B.; Green, M. A. $655 \mathrm{mV}$ open - circuit voltage, 17.6\% efficient silicon MIS solar cells. Appl. Phys. Lett. 1979, 34, 790793.

(12) Green, M. A.; Blakers, A. W. Advantages of metal-insulatorsemiconductor structures for silicon solar cells. Sol. Cells 1983, 8, 316.

(13) Ji, L.; McDaniel, M. D.; Wang, S.; Posadas, A. B.; Li, X.; Huang, H.; Lee, J. C.; Demkov, A. A.; Bard, A. J.; Ekerdt, J. G.; et al. A siliconbased photocathode for water reduction with an epitaxial $\mathrm{SrTiO}_{3}$ protection layer and a nanostructured catalyst. Nat. Nanotechnol. 2015, 10, 84-90.

(14) Hill, J. C.; Landers, A. T.; Switzer, J. A. An electrodeposited inhomogeneous metal-insulator-semiconductor junction for efficient photoelectrochemical water oxidation. Nat. Mater. 2015, 14, 11501155.

(15) Pourbaix, M. Atlas of Electrochemical Equilibria in Aqueous Solutions, 2nd ed.; National Association of Corrosion Engineers: Houston, TX, 1974.

(16) Bullock, J.; Zheng, P.; Jeangros, Q.; Tosun, M.; Hettick, M.; Sutter-Fella, C. M.; Wan, Y.; Allen, T.; Yan, D.; Macdonald, D.; et al. Lithium Fluoride Based Electron Contacts for High Efficiency n-Type Crystalline Silicon Solar Cells. Adv. Energy Mater. 2016, 6, 1600241.

(17) Wan, Y.; Samundsett, C.; Bullock, J.; Allen, T.; Hettick, M.; Yan, D.; Zheng, P.; Zhang, X.; Cui, J.; McKeon, J.; et al. Magnesium Fluoride Electron-Selective Contacts for Crystalline Silicon Solar Cells. ACS Appl. Mater. Interfaces 2016, 8, 14671-14677.

(18) Wan, Y.; Samundsett, C.; Bullock, J.; Hettick, M.; Allen, T.; Yan, D.; Peng, J.; Wu, Y.; Cui, J.; Javey, A. Conductive and Stable 
Magnesium Oxide Electron-Selective Contacts for Efficient Silicon Solar Cells. Adv. Energy Mater. 2017, 7, 1601863.

(19) Schmidt, J.; Titova, V.; Zielke, D. Organic-silicon heterojunction solar cells: Open-circuit voltage potential and stability. Appl. Phys. Lett. 2013, 103, 183901.

(20) Zhang, Y.; Zu, F.; Lee, S. T.; Liao, L.; Zhao, N.; Sun, B. Heterojunction with organic thin layers on silicon for record efficiency hybrid solar cells. Adv. Energy Mater. 2014, 4, 1300923.

(21) Greiner, M. T.; Helander, M. G.; Tang, W.-M.; Wang, Z.-B.; Qiu, J.; Lu, Z.-H. Universal energy-level alignment of molecules on metal oxides. Nat. Mater. 2012, 11, 76-81.

(22) Battaglia, C.; de Nicolás, S. M.; De Wolf, S.; Yin, X.; Zheng, M.; Ballif, C.; Javey, A. Silicon heterojunction solar cell with passivated hole selective MoOx contact. Appl. Phys. Lett. 2014, 104, 113902.

(23) Battaglia, C.; Yin, X.; Zheng, M.; Sharp, I. D.; Chen, T.; McDonnell, S.; Azcatl, A.; Carraro, C.; Ma, B.; Maboudian, R.; et al. Hole selective MoO x contact for silicon solar cells. Nano Lett. 2014, 14, 967-971.

(24) Bivour, M.; Temmler, J.; Steinkemper, H.; Hermle, M. Molybdenum and tungsten oxide: High work function wide band gap contact materials for hole selective contacts of silicon solar cells. Sol. Energy Mater. Sol. Cells 2015, 142, 34-41.

(25) Bullock, J.; Cuevas, A.; Allen, T.; Battaglia, C. Molybdenum oxide MoOx: A versatile hole contact for silicon solar cells. Appl. Phys. Lett. 2014, 105, 232109.

(26) Geissbühler, J.; Werner, J.; Martin de Nicolas, S.; Barraud, L.; Hessler-Wyser, A.; Despeisse, M.; Nicolay, S.; Tomasi, A.; Niesen, B.; De Wolf, S.; et al. $22.5 \%$ efficient silicon heterojunction solar cell with molybdenum oxide hole collector. Appl. Phys. Lett. 2015, 107, 081601.

(27) Zhang, X.; Wan, Y.; Bullock, J.; Allen, T.; Cuevas, A. Low resistance Ohmic contact to p-type crystalline silicon via nitrogendoped copper oxide films. Appl. Phys. Lett. 2016, 109, 052102.

(28) Chen, L.; Yang, J.; Klaus, S.; Lee, L. J.; Woods-Robinson, R.; Ma, J.; Lum, Y.; Cooper, J. K.; Toma, F. M.; Wang, L.-W.; et al. pType Transparent Conducting Oxide/n-Type Semiconductor Heterojunctions for Efficient and Stable Solar Water Oxidation. J. Am. Chem. Soc. 2015, 137, 9595-9603.

(29) Kenney, M. J.; Gong, M.; Li, Y.; Wu, J. Z.; Feng, J.; Lanza, M.; Dai, H. High-Performance Silicon Photoanodes Passivated with Ultrathin Nickel Films for Water Oxidation. Science 2013, 342, 836840.

(30) Sun, K.; Park, N.; Sun, Z.; Zhou, J.; Wang, J.; Pang, X.; Shen, S.; Noh, S. Y.; Jing, Y.; Jin, S.; et al. Nickel oxide functionalized silicon for efficient photo-oxidation of water. Energy Environ. Sci. 2012, 5, 78727877.

(31) Chen, Y. W.; Prange, J. D.; Dühnen, S.; Park, Y.; Gunji, M.; Chidsey, C. E. D.; McIntyre, P. C. Atomic layer-deposited tunnel oxide stabilizes silicon photoanodes for water oxidation. Nat. Mater. 2011, $10,539-544$.

(32) Hu, S.; Shaner, M. R.; Beardslee, J. A.; Lichterman, M.; Brunschwig, B. S.; Lewis, N. S. Amorphous $\mathrm{TiO} 2$ coatings stabilize $\mathrm{Si}$, $\mathrm{GaAs}$, and $\mathrm{GaP}$ photoanodes for efficient water oxidation. Science 2014, 344, 1005-1009.

(33) Allen, T. G.; Bullock, J.; Jeangros, Q.; Samundsett, C.; Wan, Y.; Cui, J.; Hessler-Wyser, A.; De Wolf, S.; Javey, A.; Cuevas, A. A Low Resistance Calcium/Reduced Titania Passivated Contact for High Efficiency Crystalline Silicon Solar Cells. Adv. Energy Mater. 2017, 7, 1602606.

(34) Avasthi, S.; McClain, W. E.; Man, G.; Kahn, A.; Schwartz, J.; Sturm, J. C. Hole-blocking titanium-oxide/silicon heterojunction and its application to photovoltaics. Appl. Phys. Lett. 2013, 102, 203901.

(35) Wang, F.; Zhao, S.; Liu, B.; Li, Y.; Ren, Q.; Du, R.; Wang, N.; Wei, C.; Chen, X.; Wang, G.; et al. Silicon solar cells with bifacial metal oxides carrier selective layers. Nano Energy 2017, 39, 437-443.

(36) Robertson, J.; Chen, C. W. Schottky barrier heights of tantalum oxide, barium strontium titanate, lead titanate, and strontium bismuth tantalate. Appl. Phys. Lett. 1999, 74, 1168-1170.
(37) Wan, Y.; Bullock, J.; Cuevas, A. Passivation of c-Si surfaces by ALD tantalum oxide capped with PECVD silicon nitride. Sol. Energy Mater. Sol. Cells 2015, 142, 42-46.

(38) Wan, Y.; Bullock, J.; Cuevas, A. Tantalum oxide/silicon nitride: A negatively charged surface passivation stack for silicon solar cells. Appl. Phys. Lett. 2015, 106, 201601.

(39) Anderson, M. D.; Aitchison, B.; Johnson, D. C. Corrosion Resistance of Atomic Layer Deposition-Generated Amorphous Thin Films. ACS Appl. Mater. Interfaces 2016, 8, 30644-30648.

(40) Li, C.; Wang, T.; Luo, Z.; Zhang, D.; Gong, J. Transparent ALD-grown $\mathrm{Ta} 2 \mathrm{O} 5$ protective layer for highly stable $\mathrm{ZnO}$ photoelectrode in solar water splitting. Chem. Commun. 2015, 51, 72907293.

(41) Chen, S.; Wang, L.-W. Thermodynamic Oxidation and Reduction Potentials of Photocatalytic Semiconductors in Aqueous Solution. Chem. Mater. 2012, 24, 3659-3666.

(42) Hu, S.; Lewis, N. S.; Ager, J. W.; Yang, J.; McKone, J. R.; Strandwitz, N. C. Thin-Film Materials for the Protection of Semiconducting Photoelectrodes in Solar-Fuel Generators. J. Phys. Chem. C 2015, 119, 24201-24228.

(43) Atanassova, E.; Spassov, D. X-ray photoelectron spectroscopy of thermal thin Ta2OS films on Si. Appl. Surf. Sci. 1998, 135, 71-82.

(44) Greiner, M. T.; Chai, L.; Helander, M. G.; Tang, W.-M.; Lu, Z.H. Transition Metal Oxide Work Functions: The Influence of Cation Oxidation State and Oxygen Vacancies. Adv. Funct. Mater. 2012, 22, $4557-4568$

(45) Imanishi, A.; Tsuji, E.; Nakato, Y. Dependence of the Work Function of $\mathrm{TiO} 2$ (Rutile) on Crystal Faces, Studied by a Scanning Auger Microprobe. J. Phys. Chem. C 2007, 111, 2128-2132.

(46) Peng, J.; Duong, T.; Zhou, X.; Shen, H.; Wu, Y.; Mulmudi, H. K.; Wan, Y.; Zhong, D.; Li, J.; Tsuzuki, T. Efficient Indium-Doped TiOx Electron Transport Layers for High-Performance Perovskite Solar Cells and Perovskite-Silicon Tandems. Adv. Energy Mater. 2017, 7, 1601768.

(47) Sinton, R. A.; Cuevas, A. Contactless determination of currentvoltage characteristics and minority-carrier lifetimes in semiconductors from quasi-steady-state photoconductance data. Appl. Phys. Lett. 1996, 69, 2510-2512.

(48) Cox, R. H.; Strack, H. Ohmic contacts for GaAs devices. SolidState Electron. 1967, 10, 1213-1218.

(49) Wan, Y.; Samundsett, C.; Yan, D.; Allen, T.; Peng, J.; Cui, J.; Zhang, X.; Bullock, J.; Cuevas, A. A magnesium/amorphous silicon passivating contact for n-type crystalline silicon solar cells. Appl. Phys. Lett. 2016, 109, 113901.

(50) Bullock, J.; Hettick, M.; Geissbühler, J.; Ong, A. J.; Allen, T.; Sutter-Fella, C. M.; Chen, T.; Ota, H.; Schaler, E. W.; Wolf, S. D. Efficient silicon solar cells with dopant-free asymmetric heterocontacts. Nature Energy 2016, 1, 15031.

(51) Yang, X.; Weber, K.; Hameiri, Z.; De Wolf, S. Industrially feasible, dopant-free, carrier-selective contacts for high-efficiency silicon solar cells. Prog. Photovoltaics 2017, 25, 896-904.

(52) Descoeudres, A.; Barraud, L.; De Wolf, S.; Strahm, B.; Lachenal, D.; Guerin, C.; Holman, Z. C.; Zicarelli, F.; Demaurex, B.; Seif, J.; et al. Improved amorphous/crystalline silicon interface passivation by hydrogen plasma treatment. Appl. Phys. Lett. 2011, 99, 123506-3.

(53) Sze, S. M.; Ng, K. K. Physics of Semiconductor Devices; John Wiley \& Sons: Hoboken, NJ, 2006.

(54) Boettcher, S. W.; Warren, E. L.; Putnam, M. C.; Santori, E. A.; Turner-Evans, D.; Kelzenberg, M. D.; Walter, M. G.; McKone, J. R.; Brunschwig, B. S.; Atwater, H. A.; et al. Photoelectrochemical Hydrogen Evolution Using Si Microwire Arrays. J. Am. Chem. Soc. 2011, 133, 1216-1219.

(55) Aberle, A. G. Overview on SiN surface passivation of crystalline silicon solar cells. Sol. Energy Mater. Sol. Cells 2001, 65, 239-248.

(56) Wan, Y.; McIntosh, K. R.; Thomson, A. F. Characterisation and optimization of PECVD SiNx as an antireflection coating and passivation layer for silicon solar cells. AIP Adv. 2013, 3, 032113. 